Ann. Biol. anim. Bioch. Biophys., I976, 16 (4), 503-520.

\title{
PUBERTÉ ET CYCLE GÉNITAL SAISONNIER DU RENARD MÂLE (VULPES VULPES)
}

\author{
M. JOFFRE \\ Centre d'Études biologiques des Animaux sauvages (C. N. R. S.) \\ et Faculté des Sciences de Poitiers \\ 79360 Beauvoir-sur-Niort (France)
}

\section{RÉSUMÉ}

L'activité spermatogénétique, les poids du testicule, de la capsule testiculaire et de l'épididyme, le poids de la prostate et la nature de ses secrétions ainsi que la testostéronémie ont été comparés au cours de la croissance testiculaire du Renard impubère et du cycle génital saisonnier du Renard adulte, libre ou captif.

Chez le Renardeau né en mars, la puberté est atteinte à l'âge de 10 mois ; elle est précédée d'une période impubère (mars à octobre) et d'une période prépubère (octobre à janvier). Chez le Renard adulte, la spermatogenèse est complète de décembre à février (période du rut). Le testicule régresse ensuite jusqu'en mai et présente une activité minimum de mai à septembre. Pendant cette période, la spermatogenèse ne dépasse pas le stade de la production de quelques spermatocytes primaires. En octobre, la spermatogenèse est à nouveau stimulée.

Chez le Renardeau, le poids de la capsule testiculaire augmente de mars à janvier. Chez l'animal adulte, lors de la régression testiculaire, la capsule croît en épaisseur et perd simultanément du poids qu'elle regagne en période de réactivation testiculaire.

Le taux de testostérone plasmatique est faible en période impubère chez le Renardeau et en période d'activité testiculaire minimum chez le Renard adulte. En novembre, alors que la spermatogenèse est déjà activée chez le jeune comme chez l'adulte, la testostéronémie croît pour atteindre un maximum en janvier ; à partir de février, elle décroît. De mai à octobre, on observe chez l'adulte, la présence de quelques pics de testostérone prouvant qu'en cette période, il existe encore une activité endocrine testiculaire.

L'épididyme et la prostate ont un poids minimum de mai à octobre chez l'adulte. Pendant cette période, 1'épididyme ne contient plus de spermatozoïdes. En période d'activité, la prostate contient du glucose et du fructose mais pas d'acide citrique ni d'ergothionéine.

Il existe des analogies importantes entre la période prépubère chez le jeune et la période de réveil testiculaire chez l'adulte, et des différences entre la période impubère et la période de repos sexuel. D'autre part, à la puberté, les poids du testicule, de la capsule, de l'épididyme et de la prostate ainsi que la quantité de testostérone secrétée sont inférieurs à ceux observés chez l'animal plus âgé, en période d'activité maximum. Enfin, nous avons constaté que la captivité n'avait pas de répercussion sur l'activité génitale de ce carnivore. 


\section{INTRODUC'TION}

Le Renard (Vulpes vulpes) est un Mammifère à activité génitale saisonnière caractérisée par une période d'involution profonde de la gonade (RowL,ANDS et PARKES, I935). Récemment (JOFFRE et JOFFRE, r973 ; JoFFRE, r973) nous avons abordé l'étude de la circulation testiculaire de ce carnivore ; ces premiers résultats nous ont amené à préciser les activités exocrine et endocrine de cette glande. En effet, 1'activité exocrine du testicule est précisée chez l'espèce Vulpes fulva et son mutant, le Renard argenté (Rowlands et PARKES, I935; VENGE, I959). Par contre, les publications consacrées à l'espèce Vulpes vulpes sont fragmentaires (RowLANDS et PARKES, I935) ou se rapportent à des animaux sacrifiés dès leur capture (FAIRLEY, I970). L'étude la plus complète a été réalisée par CREED (I960), mais elle n'aborde pas l'activité endocrine du testicule.

Nous rapportons ici les résultats relatifs à la croissance testiculaire du Renard impubère et au cycle génital saisonnier du Renard adulte sauvage capturé dans le Centre-Ouest et le Sud-Ouest de la France. Nous avons comparé les transformations liées à la période prépubère chez le jeune et celles liées à la période de réveil testiculaire chez l'adulte. En outre, nous avons cherché à savoir si les conditions de captivité dans lesquelles vivaient les renards modifiaient l'activité génitale. Dans cette analyse, les données pondérales et histologiques ont été complétées par une étude des variations de la testostérone plasmatique.

\section{MATÉRIEL E'T MÉTHODES}

Notre étude a porté sur I66 renards mâles dont 84 impubères. Ils ont été capturés de I970 à 1974 , dans des départements du Centre-Ouest et du Sud-Ouest de la France et mis en élevage dans les installations du Centre d'Étude biologiques des Animaux sauvages. Ces animaux ont été placés dans des cages individuelles, sous un abri leur assurant un photopériodisme naturel et une température ambiante voisine de la température extérieure. Afin d'étudier l'influence de la captivité sur la croissance testiculaire du Renardeau et sur le cycle génital saisonnier de l'adulte, ces animaux ont été répartis en 3 lots : animaux pubères et impubères sacrifiés dès leur capture (I), animaux sacrifiés après un certain temps de captivité et arrivés au laboratoire soit impubères (2), soit pubères (3). Les prélèvements sont effectués sur l'animal anesthésié ou sacrifié après injection intracardiaque de nembutal. Après avoir déterminé les poids des testicules, des épididymes et des prostates, ces organes ont été fixés au Bouin-Hollande ou congelés. L'état de la spermatogenèse et de la glande interstitielle du testicule a été contrôlé sur des coupes colorées à l'HemalunPicro-Indigo-Carmin. Le diamètre des tubules séminifères et l'épaisseur de la capsule ont été déterminés avec un micromètre oculaire sur 20 sections histologiques.

Nous avons recherché dans la prostate la présence de fructose (Roe, I934), de glucose (Technique de la glucose-oxydase-Merkotest), d'acide citrique (LindNer et MaNs, I96o) et d'ergothionéine (HUNTER, I950).

Enfin, nous avons déterminé le taux de testostérone plasmatique sur du sang prélevé dans la veine radiale chez des renards impubères et pubères anesthésiés. Les prélèvements ont été effectués soit tous les mois, chez certains animaux, soit uniquement au sacrifice chez d'autres. La testostérone a été dosée selon la technique radio-immunologique de LEYMARIE, STRAUSS et SCHOLLER (I974).

En général, les données sont trop peu nombreuses pour en calculer les moyennes mensuelles. Nous avons donc été amenés à considérer individuellement les animaux et à établir des courbes de variations pour lesquelles nous avons calculé les corrélations et les paramètres de régression. 
Dans quelques cas physiologiquement bien définis, nous avons exprimé les résultats par la moyenne mensuelle des valeurs, affectée de l'erreur standard sur la moyenne. Dans cẹ cas, la comparaison de deux groupes s'est faite à l'aide du test de Student.

\section{RÉSULTATS}

\section{Poids $d u$ corps (fig. I)}

Les renardeaux naissent à la mi-mars et atteignent leur poids d'adulte vers le mois d'octobre ; à cette époque, il n'est plus possible de différencier par le poids un animal impubère d'un animal adulte. La croissance du Renardeau en captivité est

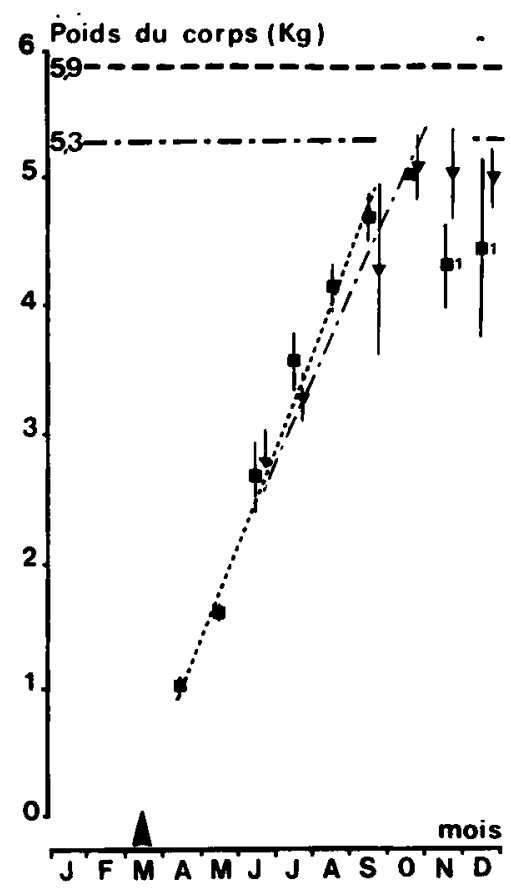

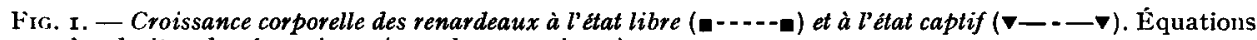
des droites de régression : ( $y$ en $\mathrm{kg}, x$ en jours)

- à l'état libre : $y=0,025 x \pm 0,259(\mathrm{P}<0,0$ I) $\} x=0$

- à l'état captif : $y=0,022 x+0,440(P<0,01)\}$ au 15 mars

$5,9 \pm 0,1$ - Poids moyen atteint par les renards adultes à l'état libre.

$5,3 \pm 0,1$ - Poids moyen atteint par les renards adultes en captivité.

Moyenne mensuelle $\pm \sigma m$.

(I) Animaux présentant à leur capture un retard de croissance. Ils ne représentent qu'un nombre faible de Renardeaux par rapport à l'ensemble des animaux capturés pendant ce mois.

peu différente de celle de l'animal en liberté. Les retards de croissance observés chez certains d'entre eux, ont été retrouvés aussi chez quelques renardeaux en liberté (fig. I). A l'état adulte, le poids moyen du Renard captif est inférieur à celui atteint 
par le Renard à l'état libre $(\mathbf{P}<0,0 I)$, mais, comme lui, il ne présente pas de cycle pondéral saisonnier. Enfin, les animaux capturés adultes perdent du poids au cours du premier mois de captivité, poids qu'ils reprennent presque toujours, par la suite.

\section{Évolution pondérale et histologique du testicule}

Évolution pondérale.

La croissance testiculaire du Renard immature est un phénomène en deux temps (fig. $2 \mathrm{~A}$ ) : à une période de croissance lente succède une période de croissance

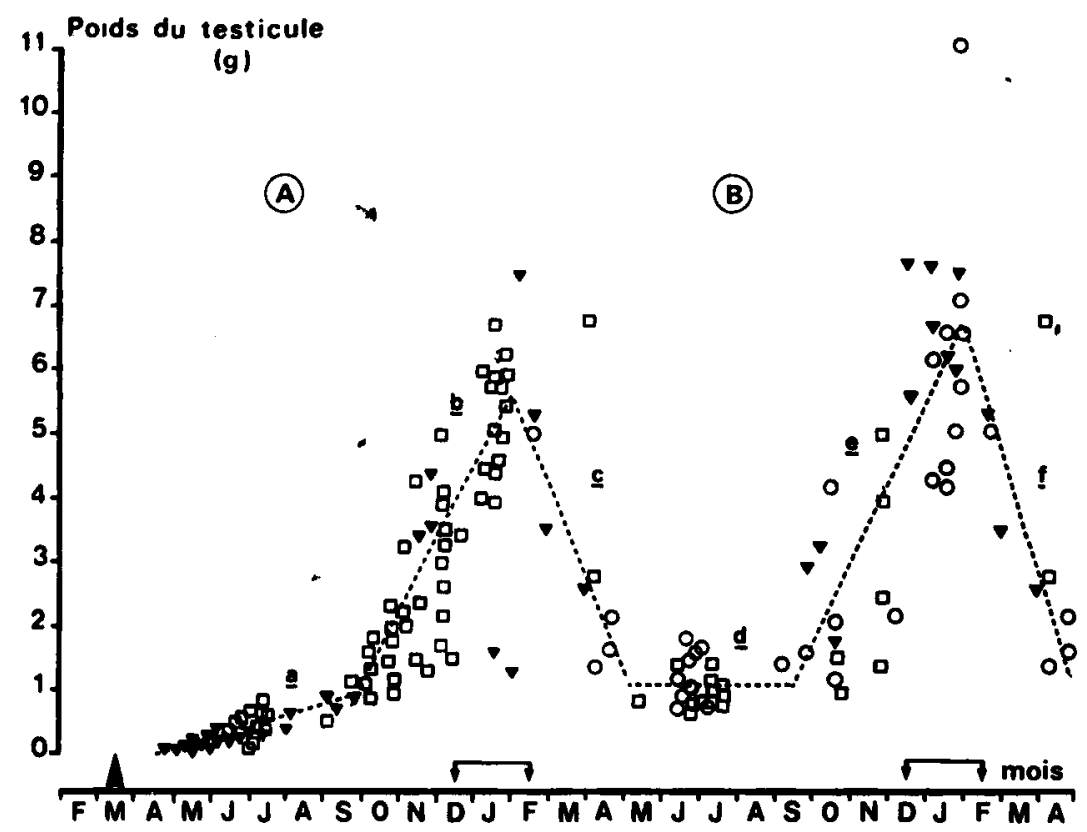

FIG. 2 A et B. - Variations saisonnières du poids du testicule chez le Renard impubère (A) et pubère (B)

Équations des droites de régression : ( $y$ en $\mathrm{g}, x$ en jours)

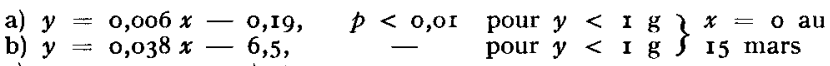
c) $\begin{array}{llll}y=-0,045 x+7, & - & - & x=0 \text { au } \\ \text { e) } y=0,043 x-9,5, & - & -\end{array}$
e) $\begin{aligned} & y=0,043 x-9,5, \\ & \text { f) } y=-0,06 \mathrm{I} x+8,4, \quad-\end{aligned}$
d) $y=\mathrm{I}, \mathrm{I} 2 \pm 0,05, n=22$

Les pentes $b$ et $e, c$ et $f$ ne sont pas significativement différentes pour $\mathrm{P}=0,05$

Légendes : Chaque valeur se rapporte à un animal

$\checkmark$ Renard sacrifié à la capture

口 Renard sacrifié après un temps de captivité-capturé impubère

- Renard sacrifié après un temps de captivité-capturé pubère

- Époque des naissances des renardeaux

$\checkmark \checkmark$ Époque du rut

rapide. Au cours de la première phase, qui s'étend en général jusqu'en octobre, le poids du testicule est en corrélation étroite avec le poids corporel $(\mathrm{P}<0, \mathrm{oI})$ et l'époque de l'année $(\mathrm{P}<0,0 \mathrm{I})$. Pendant la phase de croissance rapide, qui s'étale sur quatre 
mois environ, le poids du testicule est toujours en corrélation étroite avec l'époque de l'année $(\mathrm{P}<\mathrm{O}, \mathrm{OI})$, mais cette corrélation devient moins importante avec le poids corporel de l'animal $(\mathrm{P}=0,05)$.

Vers le mois de janvier, le testicule atteint un poids maximum. Dès lors, un phénomène cyclique s'installe (fig. $2 \mathrm{~B}$ ) : au développement maximum fait rapidement suite une période de régression qui amène le poids du testicule à une valeur minimum en mai, valeur qui reste constante jusqu'en septembre. Vers le mois d'octobre, le testicule amorce à nouveau une phase de croissance rapide, en corrélation avec l'époque de l'année $(\mathrm{P}<0, \mathrm{or})$ telle que son poids atteint une valeur maximum en janvier.

\section{Évolution histologique.}

Tous ces changements pondéraux sont liés à l'activité fonctionnelle et aux changements histologiques du testicule. Pendant la période impubère qui correspond à la phase de croissance lente du testicule, les tubules séminifères ne contiennent que deux types cellulaires : les cellules somatiques (ou de soutien) et les gonocytes (Photo I). Les cellules de soutien se répartissent à la périphérie des tubules; leur nombre par section est élevé. Les gonocytes sont volumineux et adoptent le plus souvent une position centrale dans les tubules. Les gonocytes sont le siège de nombreuses mitoses qu font que leur nombre augmente au fur et à mesure que 1'on se rapproche de la phase prépubère et que croît le diamètre des tubules séminifères (fig. 3). Tout au long de

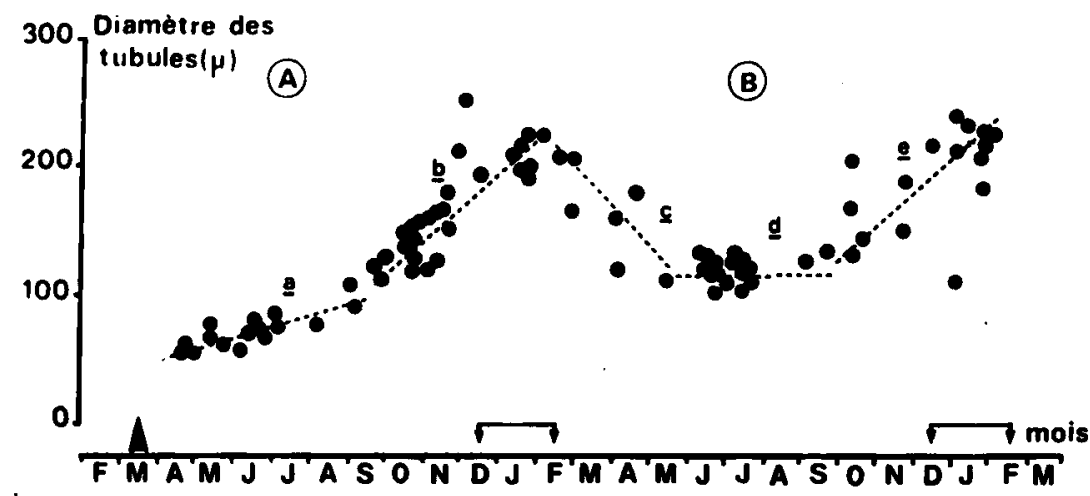

FIc. $3 \mathrm{~A}$ et B. - Variations saisonnières du diamètre des tubules séminifères chez le Renard impubère (A) et pubère (B)

Équations des droites de régression : ( $y$ en $\mu, x$ en jours)
a) $y=0,24 x+5 \mathrm{I}$,

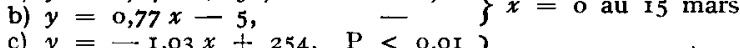
e) $\left.y=0,9 x-100, \quad \begin{array}{l}\mathrm{P}=0,01 \\ 0,05\end{array}\right\} x=0$ au $\mathrm{I}^{\mathrm{er}}$ janvier
d) $y=117 \pm 2, n=17$

Les pentes $b$ et $e$ ne sont pas significativement différentes pour $\mathbf{P}=0,05$

cette période, on observe de nombreuses cellules germinales en dégénérescence dans la lumière des tubules. On note également la présence d'un tissu intertubulaire abondant. Les cellules interstitielles sont petites, très nombreuses et déjà groupées en îlots ; certaines présentent un noyau sphérique, de diamètre égal à 5 ou $6 \mu$, avec un nucléole. 
La période prépubère correspond à la phase đe croissance testiculaire rapide. Son démarrage est marqué par la disparition des cellules en dégénérescence dans les tubules et par la position périphérique des éléments germinaux, près de la basale. On assiste ensuite à une multiplication intense des spermatogonies. Au démarrage, cette activité mitotique n'est pas visible dans toutes les sections de tubules séminifères. Ce déphasage entraîne l'apparition d'une activité cyclique de l'épithélium germinatif. Par la suite, cette activité se développe avec l'apparition des spermatocytes primaires, des spermatides et enfin des spermatozoïdes. Tout au long de cette période, on note la présence de nombreuses cellules en dégénérescence dans la lumière des tubules. Cette activité cellulaire intense s'accompagne d'un accroissement du diamètre des tubules séminifères qui passe de Ioo à 2 Io $\mu$ environ (fig. $3 \mathrm{~A}$ ). La puberté est atteinte en janvier de la première année ; le testicule produit des spermatozoïdes et secrète des androgènes.

Cet état se caractérise par la présence d'associations cellulaires comparables à ce que 1'on observe chez tous les Mammifères (photo 2). Le tissu intertubulaire a alors perdu son importance relative et les îlots de cellules de Leydig sont bien individualisés; ces cellules conservent les caractéristiques de cellules à noyau sphérique rencontrées en période impubère, mais leur noyau a un diamètre de 7 à $8 \mu$. Cette période de pleine activité est de courte durée ; il y fait rapidement suite une période de régression testiculaire. Au début de cette période (fin janvier, fin février) seulement quelques tubules présentent des cellules au noyau pycnotique, puis les signes de dégénérescence s'accentuent progressivement, et 1'on assiste à une desquamation importante de l'épithélium germinatif. La lumière du tubule est alors encombrée par des spermatozoïdes, spermatides et spermatocytes en dégénérescence ; simultanément, le diamètre des tubules décroît (fig. $3 \mathrm{~B}$ ). A la fin de cette période, les tubules contiennent des cellules de Sertoli, des spermatogonies et de nombreux spermatocytes primaires. C'est ce que l'on observe en mars-avril, alors que le testicule pèse environ $2 \mathrm{~g}$ et que le diamètre des tubules est voisin de $I_{50} \mu$. La période d'activité minimum s'étend de mai à septembre ; au cours de celle-ci, la production de spermatocytes primaires est très faible. Les tubules séminifères ont un diamètre de $120 \mu$ environ et présentent une membrane basale épaissie. A ce stade, la population cellulaire varie d'un testicule à un autre et d'une section du tubule à une autre dans un même testicule, sans qu'aucune relation puisse être établie avec le poids atteint par cette glande (tabl. I). Certaines sections contiennent quelques spermatocytes primaires ne dépassant pas le stade zygotène (Photo 3 ) ; leur nombre variable ne dépasse qu'exceptionnellement la dizaine. D'autres sections ne contiennent que des spermatogonies ; elles ont un aspect normal et se rencontrent près de la membrane basale entre les cellules de Sertoli (Photo 4). D'autres sections enfin, ne semblent contenir aucune cellule germinale' seules semblent présentes les cellules somatiques dont les noyaux adoptent alors des positions variables dans la lumière des tubules. Dans quelques cas, nous observons des cellules germinales en mitose, mais leur nombre est toujours peu élevé. Enfin, nous notons la présence de cellules germinales en dégénérescence dans la lumière de quelques tubules (Photo 3 ). Pendant cette période, le tissu interstitiel reste abondant ; certaines cellules présentent des noyaux foncés, à chromatine condensée, tandis que d'autres conservent un aspect histologique normal, identique à celui observé en période d'activité. La période de réveil testiculaire se produit le plus souvent fin septembre-début octobre et se caractérise par une activité mitotique intense, suivie 


\section{TABLEAU I}

Population en spermatocytes primaires de quelques testicules de renards en période d'activité minimum

(de mai à septembre)

\begin{tabular}{|c|c|c|c|c|}
\hline \multirow{2}{*}{$\begin{array}{l}\text { Poids du } \\
\text { testicule } \\
\text { (mg) }\end{array}$} & \multirow{2}{*}{$\begin{array}{c}\text { Diamètre } \\
\text { des tubules } \\
(\mu)\end{array}$} & \multicolumn{2}{|c|}{ Sections de tubules avec } & \multirow{2}{*}{$\begin{array}{l}\text { Nombre de } \\
\text { spermatocytes } \\
\text { par section }\end{array}$} \\
\hline & & $\begin{array}{l}\text { Cellules en dégé- } \\
\text { nérescence (1) }\end{array}$ & $\begin{array}{c}\text { Spermatocytes } \\
\text { primaires (1) }\end{array}$ & \\
\hline 900 & 114 & 6 & 5 & 1. à 2 \\
\hline 900 & 104 & 6 & 1 & 1 \\
\hline 949 & 120 & 3 & 6 & $2 \grave{a} 4$ \\
\hline 1027 & 123 & 0 & 5 & 1 à 15 \\
\hline 1064 & 117 & 0 & 16 & 1 à 8 \\
\hline 1078 & 117 & 14 & 3 & 1 \\
\hline 1118 & 120 & 8 & 4 & 1 à 3 \\
\hline 1173 & 102 & 1 & 5 & 1 à 6 \\
\hline 1445 & 128 & 3 & 6 & 1 à 7 \\
\hline 1461 & 125 & 2 & 7 & 1 \\
\hline
\end{tabular}

(1) Sur 20 sections.

(A)

(B)

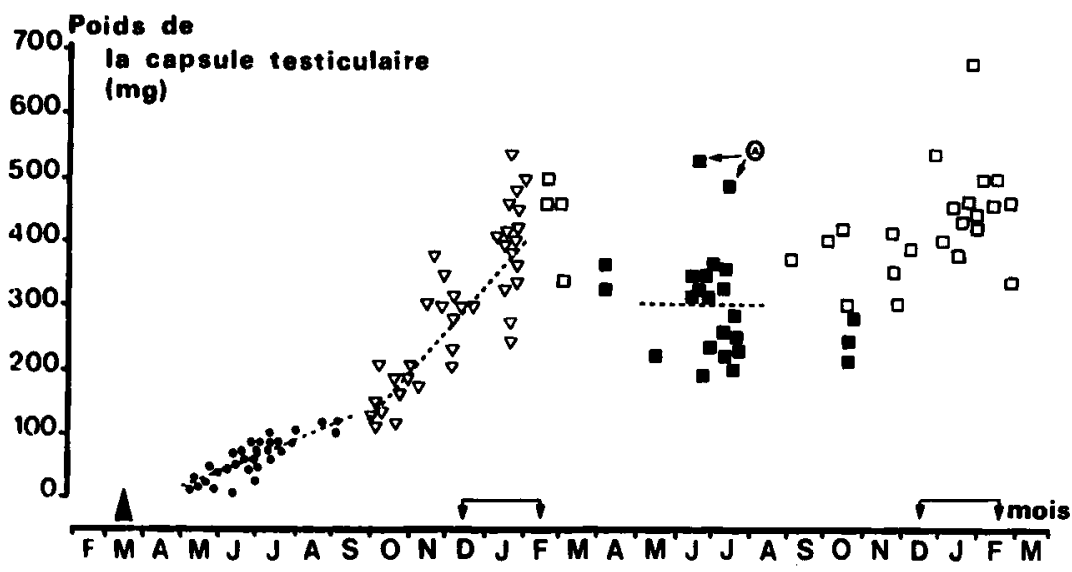

FIG. 4 A et B. - Variations saisonnières du poids de la capsule testiculaire chez le Renard impubère (A) et pubère (B)

Équations des droites de régression : ( $y$ en $\mathrm{mg}, x$ en jours)

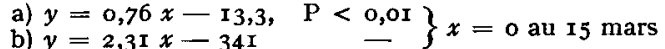

c) $y=299 \pm 19, n=24$

(A) : les testicules correspondant ont, dans ces deux cas, un poids très supérieur à la moyenne générale

- période impubère

$\nabla$ période prépubère

- activite testiculaire minimum

$\square$ testicule en activité. 
par l'apparition de nombreux spermatocytes primaires, spermatides puis spermatozoïdes. Cette période est comparable à la période prépubère chez le Renardeau et s'accompagne en particulier d'un accroissement du diamètre des tubules séminifères (fig. 3 B).

La capsule testiculaire du Renard ressemble à celle de la plupart des Mammifères ; elle émet des prolongements centripètes (les septa) qui partagent le parenchyme testiculaire en de nombreux lobules. L'épaisseur de la capsule est variable selon les zones : elle est plus épaisse à la face postérieure (accolée au corps de l'épididyme) et aux pôles (zones près de la tête et de la queue de l'épididyme) du testicule. Au cours de la croissance testiculaire chez le jeune et plus encore pendant le cycle génital saisonnier chez l'adulte, la capsule subit des variations importantes en épaisseur (50 à I50 $\mu$ chez l'animal impubère, 75 à $200 \mu$ chez l'animal prépubère, Ioo à $250 \mu$ chez l'animal en activité et 200 à $500 \mu$ chez l'animal adulte en période d'activité testiculaire minimum) La croissance de la capsule se fait en deux temps chez le Renardeau, son poids passe par un maximum en pleine activité testiculaire et par un minimum lorsque le testicule est en période d'activité minimum chez l'adulte $(\mathrm{P}<0,0 \mathrm{I})$, (fig. 4).

Évolution des effecteurs sexuels (fig. $5 \mathrm{~A}$ et $\mathrm{B}$ ).

L'épididyme de Renard présente une courbe de variations pondérales dont les caractéristiques générales sont semblables à celles du testicule (fig. 5, haut). Pendant la période impubère, l'épithélium du canal épididymaire reste bas, il est entouré d'un tissu conjonctif très développé. En période prépubère, le poids de l'épididyme augmente ; on observe simultanément un accroissement de la hauteur de l'épithélium et l'apparition de cellules germinales en dégénérescence dans la lumière du canal. Les premiers spermatozoîdes sont observés à la mi-novembre, leur nombre s'accroît jusqu'en janvier, époque à laquelle l'épididyme atteint un poids maximum. A la fin du mois de février, l'épididyme involue ; cette involution s'accompagne d'une diminution de l'épithélium et de l'apparition de nombreuses cellules en dégénérescence. En mai, 1'épididyme atteint un poids minimum qui reste constant jusqu'en septembre ; il ne contient alors plus de spermatozoïdes. En octobre, son poids s'accroît à nouveau pour atteindre un maximum en janvier.

La prostate pèse environ $3 \mathrm{~g}$ en période d'activité maximum, elle n'est plus qu'un simple bourrelet à la base de la vessie, de mai à septembre. I'examen de coupes histologiques montre qu'il y a peu de secrétions mises en réserve en période d'activité maximum ; son organisation histologique est favorable à l'hypothèse d'un écoulement permanent des secrétions. Le poids de la prostate s'accroît en période prépubère chez le jeune et en période de réveil testiculaire chez l'adulte (fig. 5, bas). La prostate est dépourvue d'acide citrique et d'ergothionéine, mais renferme du fructose et du glucose. I a teneur en glucose est surtout importante pendant les périodes de mise en activité et de mise en repos du testicule; elle est faible, voire nulle, en période d'activité maximum. La teneur en fructose ne varie pas de façon significative au cours de la période d'activité et reste souvent faible par rapport à celle du glucose. Malgré cela, le rapport fructose/glucose est toujours sıpérieur à I dans les prostates 

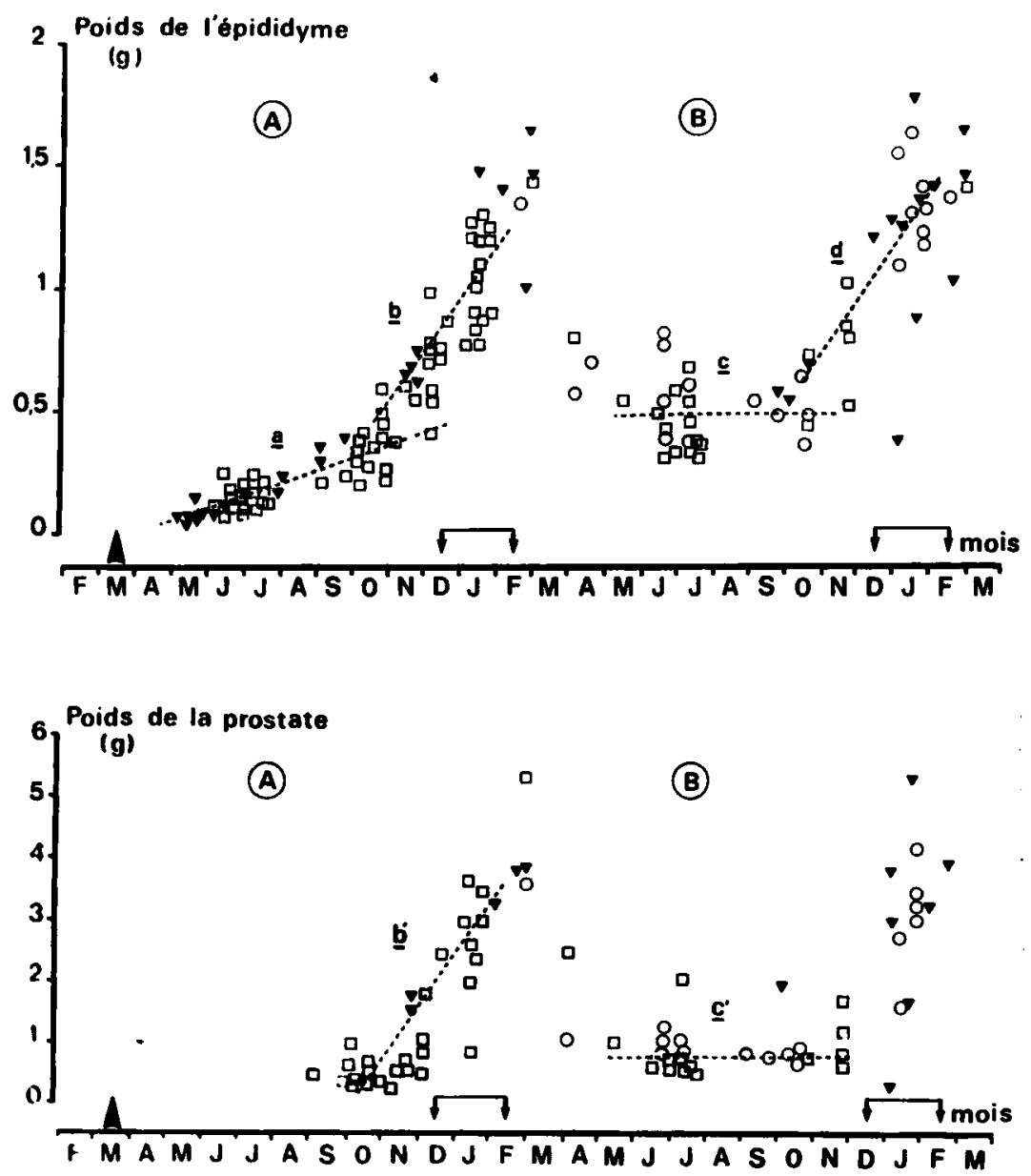

FIG. $5 \mathrm{~A}$ et B. - Variations saisonnières du poids des effecteurs sexuels chez le Renard impubère (A) et pubère (B)

$$
\begin{aligned}
& \text { en Haut: Poids de l'épididyme } \\
& \text { en Bas : Poids de la prostate. }
\end{aligned}
$$

Équations des droites de régression : ( $y$ en $\mathrm{g} x$ en jours)
$\left.\begin{array}{lll}\text { a) } y=0,002 x-0,03, & \text { P }<0,01 & \text { pour } y<0,4 \mathrm{~g} \\ \text { b) } y=0,007 x-\mathrm{I}, & \mathrm{P}<0,01 & \text { pour } y>0,4 \mathrm{~g}\end{array}\right\} x=0$ au I5 mars
b) $y=0$, or $3 x-3,7, \quad$ P $<0$, or
c) $y=0,482 \pm 0,033, n=24$
c') $y=0,839 \pm 0,099, n=15$
d) $y=0,007 x-1,5, \quad \mathrm{P}<0,01\} x=0$ au $1^{\text {er }}$ janvier 
très stimulées (période d'excrétion) et inférieur à I dans les prostates peu stimulées (période de stockage intra-tissulaire), (fig. 6).

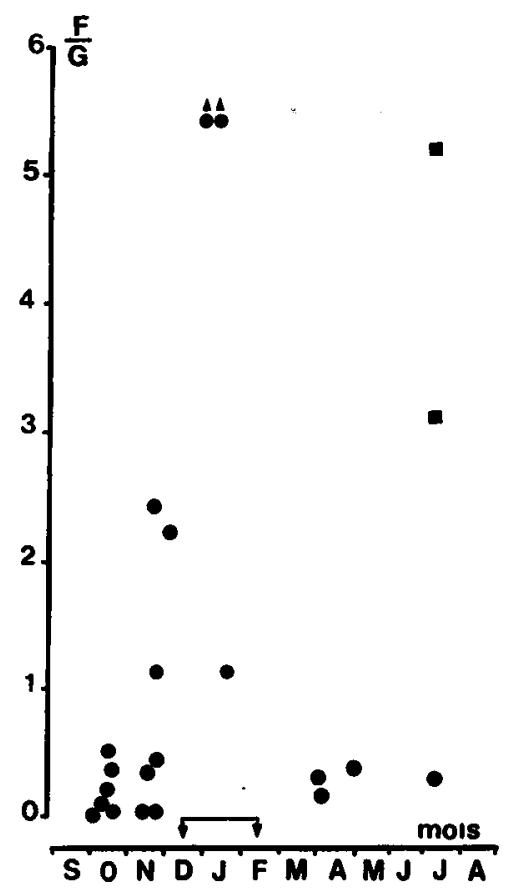

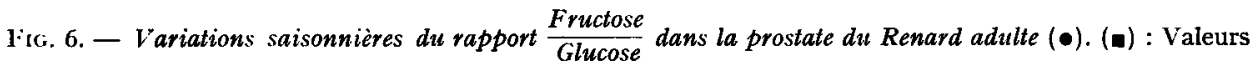
de ce rapport après stimulation de la prostate par les androgènes en période de repos sexuel (I5 mg de testostérone pendant 2 I jours). (b) : dans ces 2 cas la quantité de glucose est nulle.

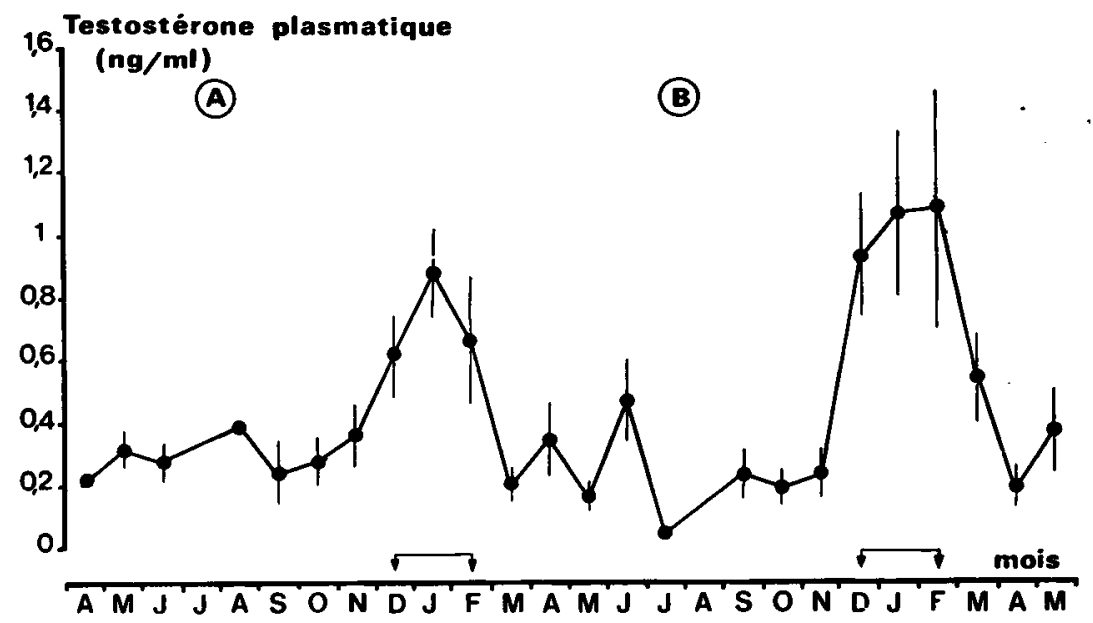

FI(;. 7 A et B. - Variations saisonnières du taux de la testostérone plasmatique du sang périphérique chez le Renard impubère A) et pubère (B)

(Moyennes mensuelles $\perp$ Frreur standard) 
La testostérone plasmatique (fig. 7). Ces résultats ont fait l'objet d'une note préliminaire (JOFFRE et JOFFRE, I975).

Chez l'animal impubère, le taux de testostérone plasmatique varie entre 0,2 et $0,4 \mathrm{ng} / \mathrm{ml}$ d'avril à novembre, puis il s'élève, passe par un maximum en janvier $(0,9 \mathrm{ng} / \mathrm{ml})$, et décroît pour atteindre en mars une valeur identique à celle du Renardeau en période impubère. Chez l'adulte, le taux est peu élevé de mars à novembre (environ $0,2 \mathrm{ng} / \mathrm{ml}$ ), il augmente rapidement, passe par un maximum de décembre à février (environ $\mathrm{I} \mathrm{ng} / \mathrm{ml}$ ) et decroît. D'octobre à mars, la secrétion d'hormones chez le jeune est environ les $3 / 4$ de celle de l'adulte (détermination faite à partir des rapports des surfaces sous les courbes). Sur quelques prélèvements effectués chez l'adulte pendant toute l'année et chez le jeune pendant la période prépubère, nous avons observé des taux de testostérone dépassant $2 \mathrm{ng} / \mathrm{ml}$ et pouvant même atteindre $35 \mathrm{ng} / \mathrm{ml}$. De telles quantités sont cependant peu fréquentes (environ 8 p. Ioo) et se rencontrent le plus souvent en période d'activité testiculaire.

\section{DISCUSSION}

Le Renard est un Mammifère qui présente une activité sexuelle en " jours courts » avec un maximum d'activité de décembre à février et un minimum de mai à septembre. Son appareil génital est semblable à celui du Chien (BOURdel, et Bressou, r953). Le Renardeau né en mars est pubère à l'âge de Io mois, ce qui corrobore les observations de Rowlands et Parkes (I935), CREED (I960), FAIRLEY (I970), faites dans les Iles Britanniques. Par ailleurs, nos observations, en accord avec celles de L, YYOD et ENGLUND (I973) confirment que le cycle génital saisonnier du Renard est modifié par la latitude de son biotope. En effet, les renards de France, comme ceux de Suisse sont matures un mois environ avant ceux des Iles Britanniques et bien avant ceux des Pays Scandinaves.

a) La croissance testiculaire du Renard impubère est semblable à ce que l'on rencontre chez la plupart des espèces (Courot, HocherEAU-de-REviERs et ORTAVANT, I970) ; son étude confirme l'existence dans le tubule séminifère de deux lignées cellulaires bien distinctes, l'une germinale et l'autre somatique. Pendant la phase prépubère, le testicule présente les mêmes phénomènes que ceux déjà mentionnés chez le Rat, la Souris, l'Agneau, le Taureau (Couror, Hochereau-de-Reviers et Ortavant, I970). En particulier, c'est au démarrage de la spermatogenèse que celle-ci s'organise en associations cellulaires identiques à ce que l'on observe chez l'adulte. Comme chez l'Agneau (Courot, I962; SkINNER et al., I968), il est d'ailleurs possible de relier chaque étape du développement de la spermatogenèse au poids du testicule (tabl. 3).

Le Renard adulte se différencie des Mammifères domestiques par l'existence d'une période d'involution profonde du testicule (tabl. 2); ce dernier point est d'ailleurs une caractéristique des Mammifères sauvages. La présence de quelques spermatocytes primaires en période d'involution maximum place le Renard au même rang que le Cerf (LINCoLN, I97I), le Putois (AUDy, I974), le Furet (IshidA, I968) et la Taupe (Allasison, I963), près du Chevreuil (Short et ManN, I966), de 
TABLEAU 2

Caractéristiques générales du cycle génital du Renard mâle

\begin{tabular}{|c|c|c|c|c|}
\hline & $\begin{array}{c}\text { Activité } \\
\text { maximum } \\
\text { (janvier } 1^{\text {re }} \text { année) }\end{array}$ & $\begin{array}{c}\text { Activité } \\
\text { minimum } \\
\text { (mai à septembre) }\end{array}$ & $\begin{array}{c}\text { Activité } \\
\text { maximum } \\
\text { (janvier } 2^{\mathrm{e}} \text { année) }\end{array}$ & $\begin{array}{l}\text { Signification } \\
\text { de la } \\
\text { différence }(\mathbf{I})\end{array}$ \\
\hline $\begin{array}{c}\text { Testicule } \\
\text { (mg) }\end{array}$ & $\begin{array}{c}5358 \pm 207 * \\
(16)\end{array}$ & $\frac{1122 \pm 5 t}{(22)}$ & $\begin{array}{c}6759 \pm 350 \\
(19)\end{array}$ & $\mathrm{P}<0,01$ \\
\hline $\begin{array}{l}\text { Capsule } \\
(\mathrm{mg})\end{array}$ & $\underset{(22)}{390}$ & $\begin{array}{l}299 \pm 11 \\
(26)\end{array}$ & $\underset{(19)}{ \pm 6}$ & $\mathrm{P}<0,01$ \\
\hline $\begin{array}{l}\text { Diamètre } \\
\text { des tubules } \\
(\mu)\end{array}$ & $\underset{(8)}{213 \pm}$ & $\begin{array}{c}117 \pm 2 \\
(17)\end{array}$ & $\frac{21 t}{(12)}$ & NS \\
\hline $\begin{array}{l}\text { Épididyme } \\
(\mathrm{mg})\end{array}$ & $1061 \pm 50$ & $\begin{array}{c}482 \pm 33 \\
(24)\end{array}$ & $1342 \pm 55$ & $\mathrm{P}<0,01$ \\
\hline $\begin{array}{l}\text { Prostate } \\
\text { (mg) }\end{array}$ & $2515 \pm 255$ & $\begin{array}{l}839 \pm 99 \\
(15)\end{array}$ & $\frac{3277 \pm 2}{(13)}$ & $\mathrm{P}=0,05$ \\
\hline
\end{tabular}

( $\left.{ }^{1}\right)<$ entre les maximums de $1^{\text {re }}$ et $2^{\mathrm{e}}$ année.

() Nombre de prélèvements.

* Moyenne $\pm \sigma_{m}$.

TABLEAU 3

Spermatogenèse et poids du testicule chez le Renard prépubère et le Renard adulte en période de réactivation testiculaire

\begin{tabular}{|c|c|c|c|}
\hline & \multicolumn{2}{|c|}{$\begin{array}{l}\text { Poids moyen du testicule } \\
\qquad(\mathrm{mg})\end{array}$} & \multirow{2}{*}{$\begin{array}{l}\text { Signification } \\
\text { de la } \\
\text { différence }\end{array}$} \\
\hline & Jeune & Adulte & \\
\hline $\begin{array}{l}\text { État de repos (A) } \\
\text { ou multiplication } \\
\text { spermatogoniale (I) }\end{array}$ & $\begin{array}{l}882 \pm 62\left({ }^{1}\right) \\
(7)(I)\end{array}$ & $\begin{array}{r}1122+5_{x}^{\prime} \\
(22) \quad(\mathrm{A})\end{array}$ & $\mathbf{P} \neq 0,01$ \\
\hline $\begin{array}{l}\text { Spermatocytes } \\
\text { primaires }\end{array}$ & $1561 \pm 114$ & $\underset{(11)}{1774} 168$ & NS \\
\hline $\begin{array}{l}\text { Spermatides rondes } \\
\text { et allongées }\end{array}$ & $\begin{array}{c}3064 \pm \underset{(18)}{ \pm} 239 \\
\text { (18) }\end{array}$ & $\begin{array}{c}3532 \pm 364 \\
(7)\end{array}$ & NS \\
\hline Spermatozoïdes & $\begin{array}{c}5358 \pm 207 \\
(18)\end{array}$ & $6 \underset{(19)}{6759}$ & $P<0,01$ \\
\hline $\begin{array}{l}\text { Pente des droites } \\
\text { de régression-limites } \\
\text { calculées pour } \\
\mathrm{P}=0,05 \\
(\mathrm{mg} / \mathrm{j})\end{array}$ & $30-44$ & $31-54$ & NS \\
\hline
\end{tabular}

(1) Moyenne $\pm \sigma_{m}$.

( ) Nombre de prélèvements. 
1'Hermine (GulamuUsern et TAM, I974), de la Martre et de la Fouine, (AUdy, I974) Mammifères dont les testicules involuent plus profondément. Chez le Renard, même au stade ultime de son involution, la spermatogenèse conserve son cycle d'activité, en sont témoins les figures de mitose et les cellules en dégénérescence rencontrées dans les tubules; lors de la période de réactivation testiculaire, comme en période prépubère, il est possible de relier chaque étape du développement de la spermatogenèse au poids du testicule (tableau 3). Dans les deux cas, ce développement s'effectue selon des modalités semblables, mais chez l'animal adulte, le démarrage se produit $x_{5}$ jours à un mois avant celui des jeunes.

On admet généralement qu'à la puberté, le testicule des Mammifères n'atteint pas son développement maximum. Cette observation faite chez l'Agneau (COUROT, I962), le Taureau (Atral et Courot, I963), le Daim (Chapman et Chapman, I970; Chaplin et White, r972), le Cerf (Lincoln, r97I a), le Blaireau (Audy, I974) et le Hérisson (Saboureau, communication personnelle), se retrouve chez le Renard (tabl. 2 et 3) : à la puberté, les poids du testicule, de l'épididyme et de la prostate ainsi que la secrétion de testostérone (environ les $3 / 4$ de celle de l'adulte) sont inférieurs à ceux observés chez l'animal adulte en pleine activité à partir de la deuxième année. L'épaississement de la capsule au cours de la croissance testiculaire chez le jeune ainsi qu'en période de repos sexuel chez l'adulte a déjà été observé, chez le Campagnol (CLARKE et Forsith, I964), l'Écureuil (DELOST, I966) et le Furet (ISHIDA, I968). Sa croissance pondérale au cours de la phase prépubère a été remarquée chez le Rat (DENDUChIs et MANCINI, I967) et chez 1'Agneau (COUROT, I97I), mais une diminution naturelle ou expérimentale du poids de la capsule n'a jamais été signalée. Ces variations saisonnières sont progressives et correspondent à une perte en eau et en matière organique ; le poids sec, en effet, diminue en période de repos sexuel (JoFFRE, I975).

b) Les variations pondérales et histologiques de l'épididyme et de la prostate sont conformes à ce qui est observé chez les Mammifères sauvages en général et sont synchrones de l'activité endocrine du testicule. Les faibles quantités de sucre stockées ne nous ont pas permis d'effectuer leur dosage direct dans les sécrétions prostatisques, ce qui nous a mené à doser aussi les sucres intracellulaires. Il ne nous est donc pas possible de conclure si un ou deux sucres réducteurs sont secrétés par la prostate de Renard.

Néanmoins, les fortes proportions de glucose observées en période transitoire sont favorables à l'hypothèse d'un stockage cellulaire de ce sucre, et les quantités sensiblement constantes de fructose, à une synthèse suivie d'une élimination rapide. Les résultats obtenus après stimulation de la prostate par les androgènes confirment cette hypothèse. Ceci est conforme aux résultats observés chez le Chien (MANN, r964).

c) L'activité endocrine saisonnière du testicule a été explorée chez quelques espèces domestiques : le Bélier (AtTaL, I970; Katongole, NAFtolin et ShorT, I974), le Bouc (Saumande et Rouger, I974), 1'Étalon (Berndtson, PicketT et NeTT, I974) et chez quelques espèces sauvages : le Chevreuil (ShORT et MaNN, I966), le Cerf (LINCOLN, I97I b), l'Hyrax (NEAVES, I973), le Caribou et le Renne (WhITEHEAD et McEvan, I97I), le Singe (Plan' et al., I974), le Blaireau (Audy et Bonnin-Laf- 
FARGUE, I975), et l'Hermine (GULAMHUShein et TAM, I974). En outre, l'activité endocrine au cours de la mise en place de la puberté a été étudiée chez l'Agneau (AtTAL, I970; SkINNER et al., I968; CotTA et al., I975), le Rat (RESko, FEDER et Goy, I968), le Cobaye (Morns et al., I972; RobER' et al., 1974), le Taureau (RAwLINGS, Hafs et Swanson, I972), l'Homme (Winter et Faiman, I972), et le Cerf (LINCOLN, I97I $a$ ). Les taux de testostérone plasmatique chez le Renard sont très inférieurs à ceux rencontrés chez les autres espèces. Le pic de testostérone est atteint lorsque le testicule produit des spermatozoïdes et présente un poids maximum. En outre, le taux minimum de testostérone coïncide avec une spermatogenèse très ralentie. Mais on peut constater que la testostéronémie, notamment chez l'adulte, augmente considérablement à partir du mois de novembre, alors que le testicule présente déjà une spermatogenèse stimulée. Ce phénomène bien que moins important, se retrouve également chez l'animal impubère.

Des prélèvements de sang fréquents dans la journée ont permis de mettre en évidence la libération pulsée de la testostérone chez de nombreuses espèces (KATONGOLE, NAFTOLIN et SHORT, I97I, I974). Le fait que chez le Renard, certains pics de testostérone soient obtenus au hasard des prélèvements, laisse envisager une libération pulsée de l'hormone, aussi bien en période d'activité maximum qu'en période d'activité minimum. La présence d'une activité endocrine en période de "repos " testiculaire confirme et complète les données histoenzymologiques de Gulamhusein et TAM (I974) chez l'Hermine. Elle corrobore les observations histologiques faites sur la glande interstitielle du Renard et met en évidence qu'en période d'activité minimum une activité spermatogénétique faible est associée à une sécrétion de testostérone. Ce dernier point suffit à rejeter l'hypothèse d'une dissociation diastématospermatique en période d'activité minimum chez cette espèce; des études sur l'hypophyse de Renard (Bugnon et Lenys, I966, I97I), que notre expérimentation corrobore sur de nombreux points, en avaient en effet laissé supposer l'existence (BUGNON et al., I975).

d) A de rares exceptions près, la captivité n'a pas de répercussion sur la croissance pondérale de l'animal impubère, elle ne modifie pas non plus son activité testiculaire. Cependant, cette période impubère est anormalement prolongée au-delà du mois d'octobre chez quelques animaux captifs. Mais un tel phénomène se retrouve dans la nature et dans les deux cas un poids corporel extrêmement faible leur est associé. Il ne faut donc pas voir là une action propre à la captivité, mais la conséquence soit d'une naissance tardive, soit d'un sevrage trop précoce. C'est au cours de la période prépubère que le Renard captif accuse un retard de croissance par rapport à son congénère vivant en liberté ; mais pendant cette période, le poids du testicule apparaît moins dépendant du poids corporel de l'animal. La captivité n'a pas de répercussion sur le poids corporel du Renard arrivé adulte ; elle n'en a pas non plus sur le déroulement du cycle génital. De ce point de vue, le Renard mâle en captivité se différencie de sa femelle; chez celle-ci en effet, la captivité entraîne un retard de 2 mois environ dans l'apparition de l'œestrus (SErTon, r9Io, in : Rowlands et Parkes, I935 ; Mondain-Monval, Dutourne, et Schol, 


\title{
REMERCIEMENTS
}

Je remercie le professeur R. Scholler, directeur de la Fondation de Recherche en Hormonologie (Fresne, 94260) pour la fourniture généreuse d'anticorps, ainsi que Mme J. JoFfRF, pour les précieux conseils qu'elle a bien voulu me prodiguer lors du dosage de la testostérone par radio-immunologie.

Ce travail fait l'objet d'un chapitre de la Thèse de Doctorat d'État ès Sciences No A.O. ro 796.

\section{SUMMARY}

\author{
PUBERTY AND SEASONAL, SEXUAL, CYCI,E \\ OF THE WILD MALE RED FOX (VULPES VULPES)
}

Spermatogenetic activity, weights of testis, testicular capsule, epididymis and prostate, as well as prostate secretions and plasma testosterone levels, are compared in the wild or captive red fox. These criteria are studied during the impubertal and prepubertal periods of the cub and during the seasonal sexual cycle of the adult.

$\mathrm{r}$. Puberty occurs in January in the cub born in March. Testicular weight increases slowly from March to October (impubertal period), then rapidly from October to January (prepubertal period). In the adult, spermatogenesis is plentiful from December to January (mating season); testis weight then increases until May and remains in quiescence until September. During this period of rest, spermatogenetic activity decreases; only a few primary spermatocytes are present in the seminiferous tubules. From October to January, testicular weight increases together with spermatogenetic activity.

2. The weight of the cub testicular capsule increases slowly from March to October, and then quickly until January. During the regressive period in the adult, the capsule becomes thicker, while its weight decreases. Testicular capsule weight again augments during the period of testicle reactivation.

3. Plasma testosterone level is low throughout the impubertal period in the cub and during the resting period in the adult. In November, plasma testosterone level increases in both groups, while spermatogenetic activity remains low. It reaches a maximum value in January, then diminishes from February to May. However, plasma testosterone peaks are observed in the resting adult.

4. Cub epididymal and prostatic weights increase slowly until October, and then rapidly between October and January; both are lower from May to October in the adult. During this period, no spermatozoa are present in the epididymis. Finally, during the mating season fructose and glucose are found in the prostate; there is no citric acid and ergothionein.

The reactive period in the adult seems very similar to the prepubertal phase in the cub; the interval of adult rest appears very different from the impubertal period. On the other hand the weights of testis, capsule, epididymis and prostate, as well as plasma testosterone level, are lower in the pubescent than in the adult fox at mating time. Captivity seems to have no effect on the genital activity of the male red fox.

\section{RÉFÉRENCES BIBLIOGRAPHIQUES}

Allanson M., I963. The reproductive tract of prepubertal and mature male moles during the anoestrus. J. Endocr., 26, IX-X.

AtTal J., 1970. Mesure des cestrogènes et des androgènes testiculaires et plasmatiques dans l'espèce ovine par des microméthodes de chromatographie en phase gazeuse : infuence de l'âge, de la saison et du cycle diurne. Thèse, Paris, C. N. R. S. no A.O. 4 I 45 .

Atral J., Courot M., I963. Développement testiculaire et établissement de la spermatogenèse chez le Taureau. Ann. Biol. anim. Bioch. Biophys., 3, 2 I9-242. 
Audy M.C., I974. Étude morphologique et expérimentale de la fonction génitale mále des Mustelidés européens. Thèse, Bordeaux, C. N. R. S., no A.O. 10048.

Audy M. C., Bonnin-Laffargue M., I975. L'activité endocrine du testicule chez le Blaireau européen (Meles meles). Arch. Biol., 86, 223-232.

Berndtson W. E., Picket B. W., Netr T.M., I974. Reproductive physiology of the stallion, IV. Seasonal changes in the testosterone concentrations of peripheral plasma. J. Reprod. Fert., 39' I I 5-I I 8 .

Bourdelle E., Bressou L., 1953. In Grasse P. P., 1969, Traité de Zoologie, Anatomie, Systématique, Biologie, 16, Masson et Cie Éditeurs.

Bugnox C., Leny's D., I966. Étude cytologique de l'adénohypophyse du Renard. C. R. Soc. Biol., Paris, 160, 2439-2442.

Bugnon C., Leny's D., I97r. Essai de caractérisation des cellules gonadotropes dans l'adénohypophyse du Renard (Vulpes vulpes). III Entretiens de Chize : Fonction gonadotrope et rapports hypotalamohypophysaires chez les animaux sauvages. Masson et Cie Éditeurs.

Bugnon C., Lenys D., Joffre M., FellmanN D., 1974. Étude cyto-immunologique des variations des cellules à prolactine et des cellules gonadotropes au cours du cycle sexuel annuel dans l'adénohypophyse du Renard mâle. C. R. Acad. Sc., Paris, 279, 289-292.

Chaplis R. E., White R. W. G., I972. The influence of age and season on the activity of the testis and epididymis of the fallow deer ; Dama dama. J. Reprod. Fert., 30, 36r-369.

Chapmay D. I., Chapman N.G., I97o. Preliminary observations on the reproductive cycle of male fallow deer (Dama dama). J. Reprod. Fert., 21, I-8.

Clarke J. R., Forsyth I. A., 1964. Seasonal changes in the gonads and accessory reproductive organs of the vole (Microtus agrestis). Gen. Comp. Endocrinol., 4, 233-242.

Cotta Y., Terqui M., Pelletier J., Courot M., 1975. Testostérone et LH plasmatiques chez l'Agneau de la naissance à la puberté. C. R. Acad. Sci., Paris, 280, 1473-1476.

Courot M., I962. Développement du testicule chez l'Agneau. Établissement de la spermatogenèse. Ann. Biol. anim. Bioch. Biophys., 2, 25-42.

Courot M., I97I. Établissement de la spermatogenèse chez l'Agneau (Ovis aries). Étude expérimentale de son contrôle gonadotrope; importance des cellules de la lignée sertolienne. Thèse, Paris, C. N. R. S. $\mathrm{n}^{\circ} \mathrm{A} . \mathrm{O} 63 \mathrm{I7}$.

Courot M., Hochereat-de-Reviers M. T, Ortavant R., I97o. Spermatogenesis. In the Testis A. D. Johnsos, W. R. Gomes, N. L. Van Demark. Ed., 1, 339-442, Academic Press, New York and London.

CREed R. F.S., 1960. Observations on reproduction in the wild red Fox (Vulpes vulpes L.). An account with special reference to the occurrence of fox dog crosses. Brit. Vet. J., 116, 419-426.

Davis J. R., Langford G. A., Kirby P. J., I97o. The testicular capsule. In the Testis, A. D. JohNson, W. R. Gomes, N. L. Van Demark. Ed., 1, 28I-337. Academic Press, New York and London.

Delost P., r966. Reproduction et cycles endocriniens de l'Écureuil. Arch. Sci. Physiol., 20, 425-456.

Dendechis B., Mancini R. E., I 967 . Quantitative variations in total and soluble collagene in testes of prepubertal and adult rats. Endocrinology, 80, $1163-1167$.

FAIRLEY J.S., r97o. The food, reproduction form, growth and development of the Fox (Vulpes vulpes), in North-East Ireland. Proc. Roy. Ir. Acad., 69, Section B, I03-137.

Gulamhusein A. P., Tam W. H., 1974. Reproduction in the male stoat (Mustella erminea). J. Reprod. Fert., 41, 303-312.

HUNTER, 1951. Ergothionein in blood and diazo reacting substance in maize. Biochem. J., 48, 265-70.

Ishid K., 1968. Age and seasonal changes in the testis of the Ferret. Arch. Histol. Jap., 29, 193-205.

JoFFre J., JOFFre M., I973. Seasonal changes in the testicular blood flow of seasonally breeding mammals : Dormouse, (Glis glis), Ferret (Mustella furo) and Fox (Vulpes vulpes). J. Reprod. Fert. 34, 227-233.

JoFFre M., 1973. Variations du débit sanguin capillaire du testicule au cours de la phase prépubère chez le Renard mâle en captivité. C. R. Acad., Sc., Paris, D, 27y, 273I-2733.

JoFFRE M., I975. Variations saisonnières du poids et du taux de collagène total de la capsule testiculaire du Renard (Vulpes vulpes) impubère et pubère. C. R. Soc. Biol. (en préparation).

Joffre M., Jofrre J., I975. Variations de la testostéronémie au cours de la période prépubère du Renardeau et au cours du cycle génital saisonnier du Renard mâle adulte (Vulpes vulpes) en captivité. C. R. Acad., Sci., Paris, D, 281, 819-821.

Katongole C. B., Naftolin F., Short R. V., i97r. Relationship between blood levels of luteinizing hormone and testosterone in bulls, and the effects of sexual stimulation. J. Endocr., 50, 457-466.

Katongole C. B., Naftolin F., Short R. V., I974. Seasonal variations in blood luteinizing hormone and testosterone levels in rams. J. Endocr., 60, Ior-Io6.

Leymarie P., Strauss N., Scholler R., I974. Dosage radio-immunologique rapide de la testostérone plasmatique chez l'adulte et l'enfant. Vérification de la spécificité par dosage en spectrométrie de masse. Path. Biol., 22, 877-882.

Lincoln G. A., I97I a. Puberty in a seasonally breeding male, the red deer stag (Cervus elaphus). J. Reprod. Fert., 25, 4I-54. 
Lincoln G. A., I97I $b$. The seasonal reproductive changes in the red deer stag (Cervus elaphus). J. Zool., 168, I05-123.

LindNer H. R., MANN T., I960. Relationship between the content of androgenic steroids in the testis and the secretory activity of the seminal vesicles in the Bull. J. Endocr., 21, 34I-359.

Llyod H. G., Englund J., 1973. The reproductive cycle of the red fox in Europe. J. Reprod. Fert., Suppl., 18, I I9-I 30 .

MANN T., 1964. Biochemistry of semen and of the male reproductive tract. Methuen London, B.

Moins N., Rigaudiete N., Robert A., Pelardy G., Delost P., i972. Variations de la secrétion des androgenes entre le $9^{\mathrm{e}}$ et le $2^{\mathrm{e}}$ jour de la vie chez le Cobaye. J. Physiol., Paris, 65, 45 I A.

Mondain-Monval M., Dutourne B., Scholler R., 1975. Activité ovarienne au cours du cycle et de l'anostrus chez le Renard roux (Vulpes vulpes L.). Ann. Endocrin. (en préparation).

NeAves W. B., 1973. Changes in testicular Leydig cells and in plasma testosterone levels among seasonally breeding rock hyrax. Biol. Reprod., 8, 46r-466.

Plant T. H., Zumpe D., Sauls M., Michael R. P., 1974. An annual rythm in the plasma testosterone of adult male rhesus monkeys maintained in the laboratory. $J$. Endocr., 62, 403-404.

Rawlings N. C., Hafs H. D., Swanson L. V., r972. Testicular and blood plasma androgens in holstein bulls from birth through puberty. J. anim. Sci., 34, 435-440.

Resko J. A., Feder H. H., Goy R. W., I968. Androgen concentrations in plasma and testis of developing rats. J. Endocr., 40, 485 .

Robert A., Gilbertas A., Rigaudik̀re N., Delost P., I974. Variations de la testostéronémie au moment de la puberté chez le Cobaye. J. Physiol., Paris, 69, I7 I A.

RoE J. H., 1934. A Colorimetric method for the determination of fructose in blood and urine. J. Biol. Chem., 107, I5-22.

Rowlands I. W., Parkes A.S., I935. The reproductive processes of certain mammals. VIII. Reproduction in Foxes (Vulpes Spp.). Proc. Zool. Soc., London, 823-84I.

Saumande J., Rouger Y., I972. Variations saisonnières des taux d'androgènes dans le plasma de sang périphérique du Bouc. C. R. Acad., Sci., D, 274, 89-92.

Short R. V., MANN T., rg66. The seasonal cycle of a seasonally breeding mammal, the roebuck (Capreolus capreolus). J. Reprod. Fert., 12, 337-35I.

Skinner J. D., Booth W. D., Rowson L. E. A., Karg H., 1968. The postnatal development of the reproductive tract of the suffolk ram, and changes in the gonadotrophin content of the pituitary. J. Reprod. Fert., 16, 463-478.

Venge O., I959. Reproduction in the fox and mink, Anim. Breed. Abstr., 27, x 29-145.

Whitehead P. E., McEwan E. H., 1973. Seasonal variation in the plasma testosterone concentration of rein deer and Caribou. Can. J. Zool., 51, 651-658.

Winter J. S. D., FaimaN C., 1972. Pituitary-gonadal relations in male children and adolescents. Pediat. Res., 6, 126-135. 
PLANCHE I

Histologie du testicule de Renard

Coloration : Hémalun-Picro-Indigocarmin

$(\times 480)$

\section{Photo I}

Testicule de Renard impubère (juillet). Les tubules contiennent de nombreuses cellules de soutien et quelques gonocytes (G). Le tissu interstitiel est abondant et renferme des îlots de cellules de Leydig (L).

\section{Рното 2}

Testicule de Renard adulte (janvier). La spermatogenèse est complète.

\section{Pнотоs 3 et 4}

Testicules de Renards adultes pendant la période d'activité testiculaire minimale (juin).

3. - Les tubules contiennent des cellules de Sertoli (S), des spermatogonies $(\mathrm{Sg})$, et quelques spermatocytes primaires (Sc). Dans la lumière on observe des cellules germinales en dégénérescence. Le tissu interstitiel présente des cellules au noyau sombre, d'autres au noyau clair.

4. - Les tubules ne contiennent que des cellules de Sertoli (S) et des spermatogonies (Sg). Le tissu interstitiel ne contient que des cellules à noyau sombre. 

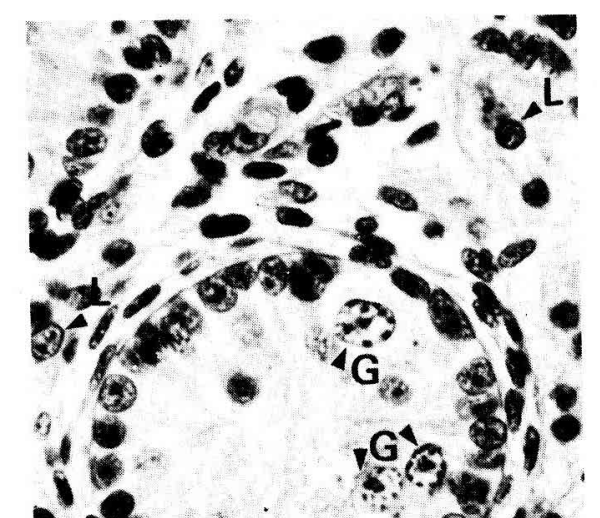

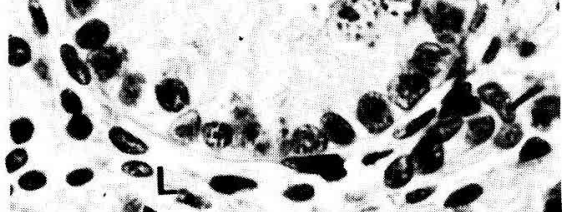

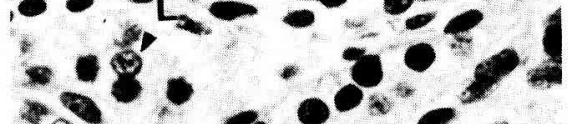

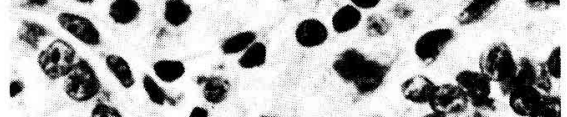

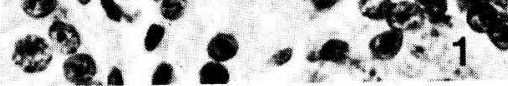

2

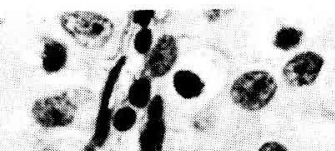

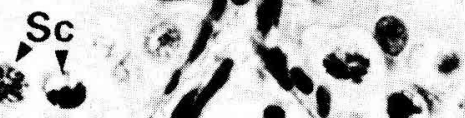

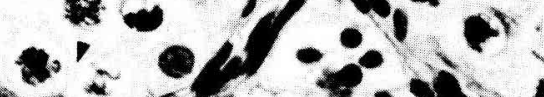

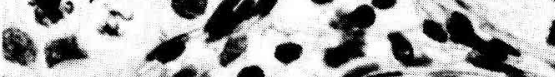

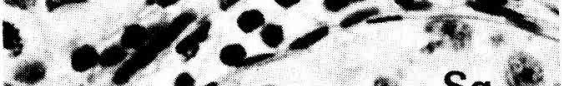

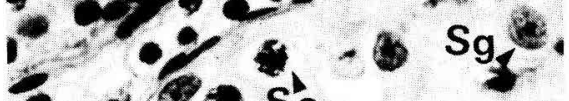
$2 \rho^{2} / 6+S^{\prime}$

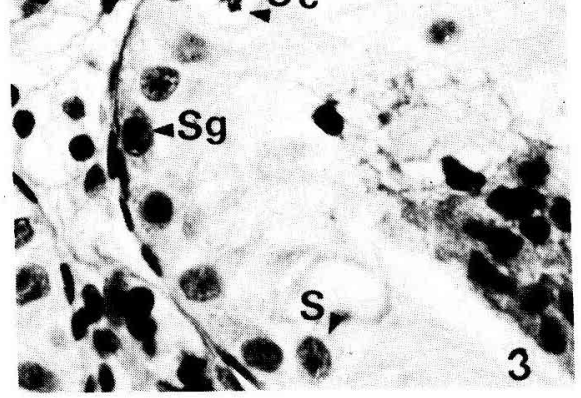

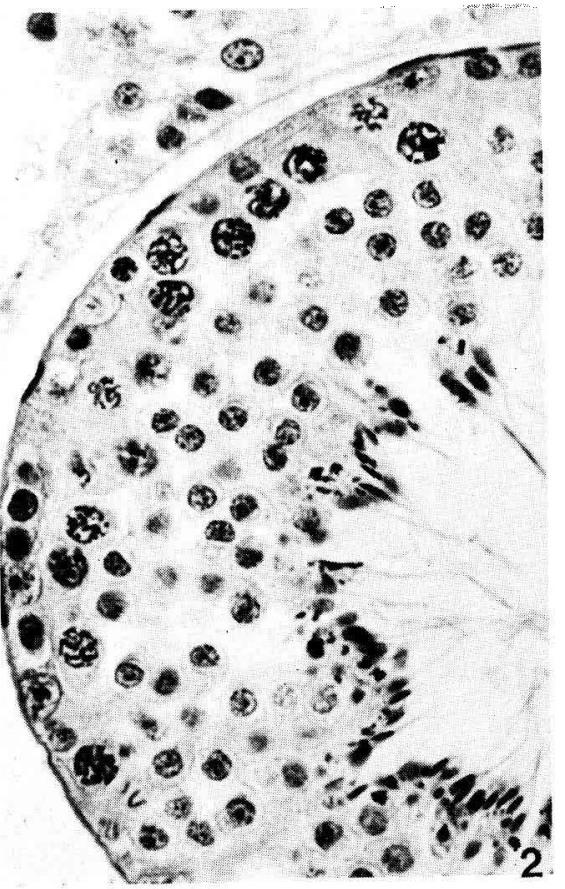

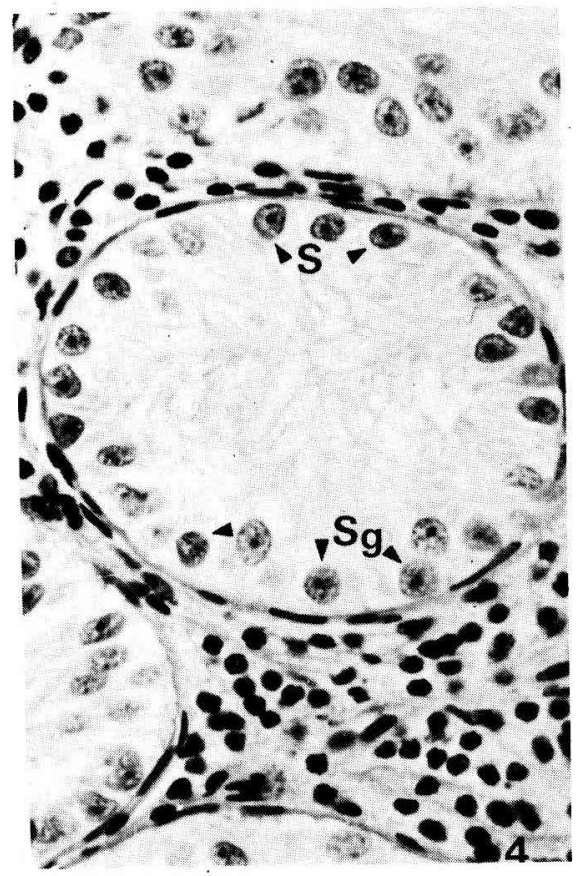

